\title{
THE DANCE OF DOOM: A RARE CASE OF EPILEPSIA PARTIALIS CONTINUA
}

\section{Major Shivkumar Gopalakrishnan ${ }^{1}$, Sangeetha Kandasamy², Sakthivel Shanmugam ${ }^{3}$}

\section{HOW TO CITE THIS ARTICLE:}

Major Shivkumar Gopalakrishnan, Sangeetha Kandasamy, Sakthivel Shanmugam. "The Dance of Doom: A Rare Case of Epilepsia Partialis Continua". Journal of Evolution of Medical and Dental Sciences 2015; Vol. 4, Issue 47, June 11; Page: 8250-8253, DOI: 10. 14260/jemds/2015/1196

ABSTRACT: BACKGROUND: "Epilepsia Partialis Continua" is considered as the status epilepticus equivalent of simple partial motor seizures. First described by Kojewnikoff in 1894, this unique type of prolonged focal seizure continues to perplex neurologists for over a century now. Controversy looms large over the site of origin of this seizure. The unequivocal cortical origin of epilepsia partialis continua is substantiated by clinical, electrophysiologic and neurosurgical evidence. However, distant, or even subcortical sites of origin have been documented earlier. Despite our progressive knowledge about this disorder, to date, neither Antiepileptic drugs (AEDs) nor invasive/surgical treatment approaches have altered the course of this disease. We herein report a case of EPC who is admitted to our hospital. CASE REPORT: On the 31 st of July, Ms A, 20/female, educated upto XII Std, was brought to the hospital for h/o 4 episodes of seizures since morning. On examination, patient was drowsy, obeyed simple commands and largely responsive. She had persistent tachycardia [110-130/mt], her BP $-110 / 80 \mathrm{mmHg}$. Neurologic examination revealed left hemiparesis and left UMN 7th nerve palsy. Her brief interictal periods [5-10mts] were punctuated by clonic seizures of left side face, upper and lower limbs [1-2mts]. She was diagnosed as "simple partial status epilepticus". Patient's blood investigations, CT and MRI brain were normal, EEG showed generalized abnormal seizure activity without any specific epileptogenic foci. Her Serum electrolytes and CSF studies including culture and biochemistry were normal. Patient was started and titrated sequentially on anticonvulsant polytherapy [Oral and injectables] with phenytoin, sodium valproate, carbamazepine, benzodiazepines, phenobarbitone, leviteracetam, clonazepam and clobazam. Her seizures remained uncontrolled with ongoing fits - day 93.

KEYWORDS: Epilepsia Partialis Continua, Simple Partial Status Epilepticus.

INTRODUCTION: "Epilepsia Partialis Continua" is considered as the status epilepticus equivalent of simple partial motor seizures. First described by Kojewnikoff in 1894, this unique type of prolonged focal seizure continues to perplex neurologists for over a century now. ${ }^{1}$ It manifests itself as focal motor clonic seizures, often persistent for days, weeks, or even years. Consciousness is preserved, since the thalamus is spared, but postictal weakness is frequently evident. ${ }^{2}$ EPC occurs due to numerous causes but ironically the manifestations of EPC is determined by the anatomical site involved rather than the pathological event causing it. It is indeed discouraging to know that our sophisticated investigation techniques and revolutionary treatment modalities have failed to tame this disorder. The natural course of EPC is very unpredictable as it marches on uninterrupted despite interventions in all but a few fortunate patients.

CASE REPORT: On the 31st of July, Ms A, 20/female, educated upto XII Std, was brought to the hospital for h/o 4 episodes of seizures since morning. Detailed history revealed that she experienced 3 episodes of focal clonic seizures on the left side of the body in the last 3 days. She also had a remote 
history of seizures 7 years ago, took treatment for 1 year and then stopped. Since then she had remained seizure free, until now. No relevant family history.

On examination, patient was drowsy, obeyed simple commands and largely responsive. She had persistent tachycardia [110-130/mt], her BP-110/80mmHg. Neurologic examination revealed left hemiparesis and left UMN 7th nerve palsy. Her brief interictal periods [5-10 mts] were punctuated by clonic seizures of left face, upper and lower limbs [1-2 mts]. During the seizures patient was fully awake and aware of her symptoms, no postictal confusion. She was diagnosed as "simple partial status epilepticus".

Her investigations revealed a $\mathrm{Hb}$ of $8.8 \mathrm{gms} \%$, blood counts were normal. Her basic blood biochemistry [Glucose, BUN, Creatinine] were within normal limits, ECG showed sinus tachycardia. Patient's CT and MRI brain were normal, EEG showed generalized abnormal seizure activity without any focal epileptogenic foci. Her Serum electrolytes [Na+131, K+ 3. 6, Cl-103, HCO3_ 23 mEq/L]. CSF studies including culture and biochemistry were uncommentable. Fundus exam was normal study.

Patient was started on anticonvulsant poltytherapy [Oral and Injectables] with phenytoin, sodium valproate, carbamazepine and benzodiazepines. Ironically patient's seizures persisted and increased in frequency and severity. Subsequently phenytoin and sodium valproate were withdrawn and phenobarbitone, leviteracetam, clonazepam and clobazam were initiated. Despite adequate dosage of anticonvulsants, her seizures remained uncontrolled though the frequency diminished. On $18^{\text {th }}$ of August she developed fever which was self-limiting, lasted for 3 days. Her blood and urine cultures did not grow pathogens. Presently the patient is still in the hospital with ongoing seizures day 93.

DISCUSSION: The estimated the prevalence of epilepsia partialis continua at less than 1 case per million population [Cockerell et al (1996)]. ${ }^{3}$ The disorder has a slight male preponderance, as shown by Sinha and Satishchandra (2007) in their review of 76 patients with epilepsia partialis continua at a tertiary care center in South India. The investigators found a male-to-female ratio of 46:30, a mean age of $30.2 \pm 23.4$ year. ${ }^{4}$

Few conditions in neurology have such varied causes as EPC. The disorder may occur due to a tumor, an abscess, syphilis, edema, embolism, or localized encephalitis. In recent times, ongoing autoimmune inflammation such as Rasmussen's encephalitis has triggered a lot of interest in the pathogenesis of EPC. Bancaud postulated a classification system for EPC wherein he divided the patients into 2 groups. Both entities start with similar seizures, but type 2 proves to be intractable and progressive.

PATHOLOGY: The term "epilepsia partialis continua" is restricted to jerks of cortical origin. Those of subcortical origin are better termed "myoclonica continua" [Cockerell et al (1996) and Shorvon (1994).2,3 The physiologic mechanism of this self-sustaining process is initiated by failure of GABAergic inhibition but is maintained by widespread potentiation of excitatory (Especially Nmethyl-D-aspartate [NMDA]) synapses. ${ }^{5}$ Another well recognized mechanism is via hyperexcitability of cortex. The cortex is endowed with powerful lateral inhibition, which prevents the spread of epileptogenic stimulus into surrounding regions. Thus EPC can go on for a long period while remaining precisely localized to a small group of muscles in a small cortical domain. 
CLINICS: EPC can affect any muscle group, however it is most common in the upper extremities. The prototype picture of clonic jerks localized to a single muscle group occurs in most patients. In exceptional cases jacksonian spread of the seizure ensues, which may even lead to a complex partial or secondarily generalized seizure. Sensory stimuli of any modality is known to modify the motor response in this type of seizure disorder. EPC can continue for long periods (Sometimes years) and our current knowledge about the determinant factors for cessation of seizures is very limited. Postictal or interictal weakness is a well-known sequelae of EPC.

MANAGEMENT: A lot of treatment modalities have been tried for this condition with discouraging results. The rarity and heterogeneity of the disorder are significant impediments to design clinical trials. In general, treatment should be aimed at correcting the primary pathology rather than the seizures themselves. Barring a few anticonvulsants, none have been shown to alter the natural history of the illness. Of note phenytoin, phenobarbitone and felbamate given orally appear to be marginally more effective than other agents. Intravenously administered valproate, diazepam and thiopental sodium are more successful, however at the cost of adverse effects. Other modalities like immunosuppression and neurosurgical techniques like subpial resections, hemispherectomy may warrant trial in extreme cases. Recently, a novel approach based on Transcranial Magnetic Stimulation may offer hope for these patients, but awaits further evaluation.

PROGNOSIS: The prognosis of epilepsia partialis continua is dictated by its underlying etiology. Most of the childhood onset EPC's are relentless and sentence the patient to gross disability or fatality. Kravljanac et al (2013) conducted a cohort study of 51 children with epilepsia partialis continua and recorded neurological consequences in $64.7 \%$ and fatal outcomes in $15.7 \%$ of the patients. ${ }^{6}$ On the contrary, in adult-onset epilepsia partialis continua, the disorder maybe self-limiting [Trauma, stroke], remediable [Cortical dysplasia, AV malformation] or progressive (e.g, tumor, carcinomatous meningitis); the outcome is determined by the primary disease.

CONCLUSION: Epilepsia Partialis Continua is a rare form of seizure disorder which involves focal muscle twitching for prolonged periods of time. It is resistant to treatment and quite disabling to the patients. The precise mechanism of initiation and persistence, as also the environmental determinants of this status epilepticus is yet to be elucidated. We herein report a case of EPC occurring in a young lady which is ongoing presently [Day 93]. It is with deep anguish that we observe this patient every day, though ironically, we find ourselves as disabled as the sufferer.

ACKNOWLEDGEMENT: We thank all the staff of Medicine Department Govt. Villupuram Medical College for their dedication and efforts put into the management of this patient and their help towards our academic purpose.

\section{REFERENCES:}

1. Vein AA, van Emde Boas W. Kozhevnikov .Epilepsy: the disease and its eponym. Epilepsia. Feb 2011; 52 (2): 212-8.

2. Gastaut H. [Semeiology of myoclonus and analytic nosology of myoclonic syndromes]. Rev Neurol (Paris). Jul 1968; 119 (1): 1-30. 


\section{CASE REPORT}

3. Cockerell OC, Rothwell J, Thompson PD, Marsden CD, Shorvon SD. Clinical and physiological features of epilepsia partialis continua. Cases ascertained in the UK. Brain. Apr 1996; 119 (Pt2): 393-407.

4. Sinha S, Satishchandra P. Epilepsia Partialis Continua over last 14 years: experience from a tertiary care center from south India. Epilepsy Res. Apr 2007; 74 (1): 55-9.

5. Mazarati AM, Wasterlain CG. Blockers of NMDA receptor restore paired-pulse inhibition in the rat dentate gyrus lesioned by perforant path stimulation. Neurosci Lett. Oct. 3 1997; 234 (2-3): 135-8.

6. Kravljanac R, Djuric M, Jovic N, Djordjevic M, Zamurovic D, Pekmezovic T. Etiology, clinical features and outcome of epilepsia partialis continua in cohort of 51 children. Epilepsy Res. Mar 2013; 104 (1-2): 112-7.

\section{AUTHORS:}

1. Major Shivkumar Gopalakrishnan

2. Sangeetha Kandasamy

3. Sakthivel Shanmugam

\section{PARTICULARS OF CONTRIBUTORS:}

1. Assistant Professor, Department of Internal Medicine, GVMC \& H, Tamilnadu.

2. Assistant Professor, Department of Biochemistry, GVMC \& H, Tamilnadu.

\section{FINANCIAL OR OTHER} COMPETING INTERESTS: None
3. Trainee, Department of Internal Medicine, GVMC \& H, Tamilnadu.

\section{NAME ADDRESS EMAIL ID OF THE CORRESPONDING AUTHOR:}

Dr. Sakthivel Shanmugam, No.10/10 Appavu Gramini $3^{\text {rd }}$ street, Mandaiveli, Chennai-600028, Tamilnadu.

E-mail: sakthivelgokulam333@gmail.com

Date of Submission: 30/04/2015.

Date of Peer Review: 05/05/2015.

Date of Acceptance: 04/06/2015.

Date of Publishing: 11/06/2015. 